\title{
Research on TD-LTE Wireless Communication Network Propagation Model Optimization and Visual Simulation Based on GIS
}

\section{Tongyan Li}

Nanjing Polytechnic Institute

Shuchun Hua

Jinling Institute of Technology

Le Kang ( $\nabla$ kangle@hbue.edu.cn )

Hubei University of Economics https://orcid.org/0000-0001-7574-9428

Sheng-Hung Chang

Hubei University of Economics

\section{Research Article}

Keywords: TD-LTE, propagation model, path loss, coverage prediction, network optimization spalling

Posted Date: April 16th, 2021

DOI: https://doi.org/10.21203/rs.3.rs-391321/v1

License: (a) (i) This work is licensed under a Creative Commons Attribution 4.0 International License. Read Full License

Version of Record: A version of this preprint was published at EURASIP Journal on Wireless Communications and Networking on July 31st, 2021. See the published version at https://doi.org/10.1186/s13638-021-02007-0. 


\title{
Research on TD-LTE Wireless Communication Network Propagation Model Optimization and Visual Simulation Based on GIS
}

\author{
Tongyan $\mathrm{Li}^{1}$, Shuchun $\mathrm{Hua}^{2}$, Le Kang ${ }^{3}$, Sheng-Hung Chang ${ }^{3}$ \\ 1. School of Economics and Management, Nanjing Polytechnic Institute, Nanjing, China \\ 2. Business School, Jinling Institute of Technology, Nanjing, China \\ 3. School of Business Administration, Hubei University of Economic, Wuhan, China \\ Corresponding Author's Email: kangle@hbue.edu.cn
}

\begin{abstract}
With the support of GIS spatial analysis technology, based on an in-depth study of the wireless propagation environment of a city, combined with the analysis of project requirements, it proposes to use the SPM model to correct the propagation model parameters, using SPM The wireless propagation model, and research and analysis of the SPM wireless propagation model correction algorithm, further corrected the parameters of a city's SPM wireless propagation model. On this basis, the propagation loss of several classic propagation models in different environments are compared, and the SPM propagation model suitable for the signal frequency band and propagation environment of this study is selected. The correction of the SPM propagation model is based on the designed correction principle and correction process, that is, the weighted least square method is used to fit and analyze the measured level data to obtain an SPM prediction improvement model with local characteristics, and according to the designed verification link Evaluation of the correction results shows that the accuracy requirements are met. Based on the corrected SPM prediction model, link loss calculations were performed on the 13 test base stations studied in the experiment, and the effective coverage radius of each base station community was obtained. In combination with GIS technology, model parameters and workers of each base station participated in the electronic map loading of the area Go to the network planning software to get the wireless signal coverage prediction map of each base station. Finally, according to the technical requirements of the TD-LTE system network planning and network optimization engineering, the objectiveness and rationality of the site selection and number of base stations in the area were verified, and specific problems regarding poor coverage and overlapping coverage in the area were proposed.
\end{abstract}

Keywords: TD-LTE; propagation model; path loss; coverage prediction; network optimization spalling

\section{Introduction}

This thesis takes TD-LTE as the research object. TD-LTE is now the most used network service, but because the technology is independently developed in China, there is no mature communication network construction experience to learn from, so TD-LTE wireless network Planning is a challenge. The first step of communication network planning is to determine the propagation model of radio wave signal coverage prediction, because the propagation model is suitable to predict the median loss of the signal from transmission to reception. Although domestic and foreign scholars have obtained many classic propagation models through experimental research, and these classic models have certain universality, the environment in specific scenes changes, which will lead to less accurate predictions. Therefore, in network planning, the path loss formula or the propagation model must be corrected to obtain more accurate propagation prediction losses, and preparations are made for subsequent coverage planning, capacity planning, and link budgeting.

In practical applications, GIS-based communication system planning, coverage forecasting, data analysis and processing, network quality monitoring and analysis, and other functions [1-3] are all kinds of communication data with geographic attributes and computer technology, The combination of GIS spatial analysis technology, visualization and simulation technology enables users to easily and intuitively see the coverage effect, scope, and obtain technical support for network planning and optimization. The necessity and importance of the introduction of GIS technology from the perspective of actual needs and application prospects [4-6], and the construction of various types of modern dedicated communication information systems based on integrated development with GIS, are provided for the management, analysis and planning of all communication resources. Brand-new methods and means; Analyzes the actual key problems of communication network resource data, and realizes the function of visual information management using GIS technology in communication network resource management [7,8]; proposes a method using drive test data A model for propagation correction, which can be applied to mobile networks of different standards and standards $[9,10]$; according to the existing wireless signal propagation prediction model, using the $\mathrm{CW}$ continuous wave test method, by improving the traditional model algorithm and correction process, Prediction of the signal coverage in a certain propagation environment and estimation of the propagation loss value of a certain distance of the signal have obtained good simulation results. It provides a strong engineering method for accurate coverage prediction of wireless signals in specific research areas [11-13]. During the widespread application of the second-generation mobile communication systems (GSM and CDMA), the research work on propagation models has developed unprecedentedly, and a propagation model has been formed. This propagation model is suitable for many different wireless propagation environments[14-15]. According to the different application 
scenarios of propagation models, they can generally be divided into three categories: macro-cell propagation models, micro-cellular propagation models, and indoor propagation models [16-19]. The application status and trends of GIS technology in the communication industry are analyzed, and the visualization of GIS and the optical fiber routing management and fault location of communication networks are combined to obtain the important value of GIS in the management of communication networks [20]; Based on a comparison of various domestic and foreign existing and semi-experienced propagation models, each model is used for simulation practice. Finally, it is given that different propagation environments should choose different LTE propagation models [21]; the use of GIS technology to obtain the research area Building height, density and other information, collected a large amount of measured data, the prediction model obtained is consistent with the actual situation, and a specific method for predicting the coverage of wireless communication signals based on the building's field information is designed. [22, 23].

According to the basic electromagnetic wave propagation theory in mobile communications, radio waves are affected by factors such as terrain, frequency, and distance. They propagate in the propagation space with path loss, slow fading loss, and fast fading. Multipaths are generated from two aspects, time and space Loss, affected by terrain and features, will produce shadow effects, near and far effects, and Doppler effects. According to the electromagnetic wave space propagation attenuation theory, there are mainly theoretical analysis methods and field measurement methods for studying the electromagnetic field propagation space field distribution of mobile communication base stations. This paper mainly uses statistical prediction models to perform signal coverage prediction. After comparing the commonly used classic outdoor macro-cell signal propagation models, the propagation model suitable for the study area is determined to be the SPM general model. This model considers the signal propagation path loss and the distribution of ground features, antenna height, carrier frequency, distance and other various The effect of parameters on the signal. Then, the received level signal is predicted by the LTE downlink budget method, and a weighted least square method is used to fit a local prediction model, and the prediction result is verified to meet the index evaluation. The coverage of each TD-LTE base station in the study area was calculated, and the prediction results of the wireless signal propagation path loss coverage of each base station were obtained. Using GIS analysis tools and network planning software, a visual simulation of the loss prediction results was achieved.

\section{Methodology}

\subsection{Classification of wireless communication signals}

Table 1 details the classification of wireless communication signals by frequency. It is Included band name, wavelength range, frequency range, propagation mode, and application occasions.

Table 1 Classification of wireless communication signals by frequency

\begin{tabular}{|l|l|l|l|l|}
\hline Band name & $\begin{array}{l}\text { Wavelength } \\
\text { range }\end{array}$ & $\begin{array}{l}\text { Frequency } \\
\text { Range }\end{array}$ & Propagation mode & Application occasion \\
\hline $\begin{array}{l}\text { Long wave } \\
\text { band }\end{array}$ & $2000-20000 \mathrm{~m}$ & $30-300 \mathrm{KHz}$ & Ground wave & $\begin{array}{l}\text { Long distance } \\
\text { communication }\end{array}$ \\
\hline Mid-band & $200-2000 \mathrm{~m}$ & $300-3000 \mathrm{KHz}$ & Ground wave & $\begin{array}{l}\text { Broadcast, communication, } \\
\text { navigation }\end{array}$ \\
\hline $\begin{array}{l}\text { Short wave } \\
\text { band }\end{array}$ & $20-200 \mathrm{~m}$ & $3-30 \mathrm{MHz}$ & Sky wave & $\begin{array}{l}\text { Middle distance } \\
\text { communication }\end{array}$ \\
\hline $\begin{array}{l}\text { Ultra-short } \\
\text { wave band }\end{array}$ & $\mathrm{I} 20 \mathrm{~m}$ & $30-300 \mathrm{MHz}$ & $\begin{array}{l}\text { Linear propagation, } \\
\text { scattering }\end{array}$ & $\begin{array}{l}\text { Mobile communication, TV } \\
\text { broadcasting }\end{array}$ \\
\hline $\begin{array}{l}\text { Decimeter wave } \\
\text { band }\end{array}$ & $200-2000 \mathrm{~m}$ & $300-3000 \mathrm{MHz}$ & $\begin{array}{l}\text { Linear propagation, } \\
\text { scattering }\end{array}$ & $\begin{array}{l}\text { Mobile communications, } \\
\text { satellite communications, }\end{array}$ \\
\hline $\begin{array}{l}\text { Centimeter } \\
\text { wave band }\end{array}$ & $\mathrm{I} 20 \mathrm{~m}$ & $3--30 \mathrm{GHz}$ & Straight propagation & TV broadcasting, radar \\
\hline $\begin{array}{l}\text { Millimeter wave } \\
\mathrm{I} \sim 20 \mathrm{~m}\end{array}$ & $30--300 \mathrm{GHz}$ & Straight propagation & $\begin{array}{l}\text { Relay communication, } \\
\text { radar, satellite }\end{array}$ \\
\hline
\end{tabular}

\subsection{TD-LTE communication data processing method}

(1) Data filtering

It is generally considered that the range between $0.1 \mathrm{R}-2 \mathrm{R}$ from the slave station is a reasonable test range, and $\mathrm{R}$ is the expected test cell radius. The strength of the test signal does not have a strict linear relationship with the propagation distance. The test distance is too close, the test data is small, and geographical averaging cannot be performed. At this time, the receiving terminal is in a critical state to resolve the signal, and its value is easily affected by transient fluctuations. In addition, some test data must be filtered from the sampling data. The specific filtering conditions are as follows:

1. Under the overhead, medium tunnel, where GPS cannot locate accurately. 
2. Data that are too close or too far away from the antenna, and data that are too close or too far away are not easy to average.

3. Data with too weak signal strength (less than $-115 \mathrm{dBm}$ )

4. Incorrect data caused by inaccurate antenna pattern

5. Data on other sections that have been determined not to meet the requirements on the $\mathrm{CW}$ test route, such as: test data for repeated sections;

6. Signal strength exceeding one $40 \mathrm{dBm}$ but unexplainable fading;

Filters of types 1 and 5 need to be completed before geographic averaging, and filters of types 2 and 3.4 need to be completed after geographic averaging of data.

(2) Data discrete processing principle

Assuming that the vehicle speed between the two data collection points is uniform, and the time difference between each two recording points is equal, the receiver data sampling speed is much faster than the GPS positioning speed. The test records cannot be directly geographically averaged; usually, these collected data are evenly distributed to the road section between the two test points in chronological order to meet a sufficient number of points per 6 meters in length.

(3) Geographic average

The purpose of geographic averaging is to eliminate fast fading and retain the effects of slow fading. There are two methods of geographic averaging:

Method 1: The entire test area is made into a grid with a side length of 6 meters, and the communication data collected in each grid is arithmetically averaged.

Method two: Separately spaced along the test route, 6 meters per segment, arithmetically average the test data collected in each segment, and uniformly select a point as the average test point.

\subsection{Analysis of wireless communication network propagation model}

The GIS-based wireless communication network propagation model uses topographic data, that is, a digital electronic map database, multiple diffraction mechanisms, taking into account the propagation conditions in different landscapes, and effective antenna heights. At the same time, the model can be corrected. The model is based on the following formula:

$$
P R=P I X-\left\{\begin{array}{l}
K 1+K 2 \lg (d)+K 3 \lg (\text { HIXeff })+K 4 * \text { Diffraction }+K 5 \lg (d) * \lg (\text { HIXeff }) \\
+K 6(\text { HRXeff })+\text { Kclutter }
\end{array} * f(\text { clutter })\right.
$$

The parameters in the formula are as follows:

PR: terminal received power $(\mathrm{dBm})$

PTx: Base Station Transmit Power (EIRP) (dBm)

$\mathrm{K} 1$ : Frequency-dependent constant (dB)

K2: Multiplier factor for $\operatorname{Lg}(\mathrm{d})$. This value indicates how fast the field strength changes with distance.

$\mathrm{d}$ : distance between transmitter and receiver $(\mathrm{m})$

K3: $\lg$ (HTxeffj multiplier factor, this value indicates the field strength changes with the antenna height

HTXeff: effective height of the transmitting antenna (m)

K4: multiplier factor for diffraction calculation, $\mathrm{K} 4$ must be a positive number

Diffraction: Diffraction loss $(\mathrm{dB})$ caused by passing an obstacle path

K5: Multiplier for Lg (TXHeff) and Lg (d)

K6: HRxeff multiplier

Hrxeff: effective height of the receiving antenna (m)

Kclutter: multiplier factor for $\mathrm{f}$ (clutter), this value represents the weight of ground loss

$\mathrm{f}$ (clutter): average weighted loss of different features

It is worth noting that the model calculates the loss $\mathrm{f}$ (clutter) caused by the ground features, which is a weight loss due to the ground features passing from the transmitter to the receiver. The weighting method is also optional.

\subsection{Optimization of wireless communication network propagation model}

The method of using this model to correct is: first set the parameter values K1-K6, usually you can choose the default value of the frequency to set it, or other similar terrain correction parameters, and then use this model for wireless propagation prediction Compare the predicted value with the drive test data, and then modify the model parameters based on the statistical results of the obtained difference. After continuous iterative processing, until the mean square error and standard deviation of the top test value and the drive test data reach the minimum, Each parameter value of the model obtained at that time is the required correction value.

The principle used in the above method is the principle of least squares, and the theory is as follows: 
Let a plane straight line (ie, one-variable regression) fit. Let the theoretical straight line be $\mathrm{y}=\mathrm{a}+\mathrm{bx}$. There are $\mathrm{n}$ sets of measured values $(\mathrm{xl}, \mathrm{yl}),(\mathrm{x} 2, \mathrm{y} 2) \ldots(\mathrm{xn}, \mathrm{yn})$, which is nearly linearly distributed in plane coordinates.

The principle of the least square method is to say that if the sum of squares of the difference between the theoretical positions of the actual measured values $\mathrm{t} 7$ is the smallest, then this theoretical straight line is the regression that best reflects the actual measured values.

$$
\Delta=\sum_{i=1}^{n}\left(y_{i}-y\right)^{2}=\sum_{i=1}^{n}\left(y_{i}-a-b x_{i}\right)
$$

Finding regression coefficients a and $\mathrm{b}$ by partial derivative method.

$$
\frac{\partial \mathrm{V}}{\partial a}=2 \sum_{i=1}^{n}\left(y_{i}-a-b x_{i}\right)=0
$$

which is:

$$
\begin{aligned}
& \sum_{i=1}^{n} y_{i}=n a+b \sum_{i=1}^{n} x_{i} \\
& \frac{\partial \mathrm{V}}{\partial b}=2 \sum_{i=1}^{n}\left(y_{i}-a-b x_{i}\right)=0 \\
& \sum_{i=1}^{n} x_{i} y_{i}=a \sum_{i=1}^{n} x_{i}+b \sum_{i=1}^{n} x_{i}^{2}
\end{aligned}
$$

Find the regression coefficients $a$ and $b$.

Let $x 1$ be the predicted value of a test point, $x 2$ be the measured value at that point, $y_{i}=x 1-x 2, \bar{y}=\frac{\sum y_{i}}{N}$ and $\bar{y}$ be the statistical average difference

$$
\begin{aligned}
& \text { RMS , Error }=\sqrt{\frac{\sum y_{i}^{2}}{N}} \\
& \text { StdError }=\sqrt{\frac{\sum\left(y_{i}-\bar{y}\right)^{2}}{N-1}}
\end{aligned}
$$

It can be seen that when $\bar{y}$ is 0 , RMS Error $=$ StdError.

The result of model optimization is to make the values of RMS Error and StdError to a minimum, and to judge the fit between the model results and the actual environment. The parameter correction algorithm for the SPM model is as follows:

First define the following parameters: $\mathrm{L}$ is the theoretical loss value; $\mathrm{L} 1$ is the actual loss value; DL $=\mathrm{L} 1-\mathrm{L}$ is the difference between the actual value and the theoretical value; $\mathrm{K} 1$ is a correction factor related to frequency f: K2: based on $\log (d)$ Correction factor. After sampling analysis of the system, a matrix $\log (d)$ of the distance $\mathrm{d}$ between the transmitter and the receiver is obtained.

$$
\lg d n=(\lg d 1, \lg d 2 \ldots \lg d n)
$$

Difference between theoretical value and actual value VLn .

$$
\mathrm{V} L n=(\mathrm{V} L 1, \mathrm{~V} L 2 \ldots \mathrm{V} L n)
$$

Define the correction factor constant: $K=(K 1, K 2)$

$$
K=\mathrm{VLn} / A
$$

\section{Optimization and visualization of TD-LTE wireless communication network model based on GIS}

\section{1 SPM propagation model optimization algorithm}

Although theoretically all K parameters of the SPM model can be corrected, in practical applications, due to various conditions during data collection, the experimental data are limited, so not all $\mathrm{K}$ coefficients can be accurately corrected. Generally speaking, The propagation distance is an important factor affecting the path loss, so correction of the coefficient $\mathrm{K}$ of $\operatorname{lgd}$ is a priority. In addition, the carrier frequency has a certain effect on the loss result, so the frequency factor K1 must also be corrected. In addition to the above two coefficients, other Kfactor correction suggestions are shown in Table 2.

Table $2 \mathrm{~K}$ value correction coefficient term of SPM model

\begin{tabular}{|l|l|l|}
\hline K value & Coefficient meaning & Whether to correct \\
\hline K1 & Constant loss, frequency dependent & Y \\
\hline K2 & Transmission distance loss & Y \\
\hline K3 & Loss factor caused by changes in base station antenna height & N \\
\hline
\end{tabular}




\begin{tabular}{|l|l|l|}
\hline K4 & Diffraction loss, only hilly terrain will be considered & $\mathrm{N}$ \\
\hline K5 & $\begin{array}{l}\text { Influence of base station antenna height and propagation distance on } \\
\text { received power }\end{array}$ & $\mathrm{N}$ \\
\hline K6 & $\begin{array}{l}\text { Gain caused by changes in antenna height at the receiving end, when the } \\
\text { terminal height is 1. 5m, usually set to 0 }\end{array}$ & $\mathrm{N}$ \\
\hline Kclutter & Various ground loss, the default value is 1 & $\mathrm{N}$ \\
\hline
\end{tabular}

Since the antenna height of each base station remains unchanged during the data acquisition process, the degree of change in the height of the base station antenna is a coefficient K3. Because the change in terrain is generally not obvious, the antenna height is considered to be unchanged, so it is recommended that K: take the default value No corrections are made. Because the area under study is flat in the suburbs or rural areas, and the difference is determined, it is not recommended to correct K4. The correlation coefficient K5 of the antenna height of the base station and the signal propagation distance is not corrected. Similar to K3, it is also recommended to take the default value without correction.

The formula of the TD-LTE path loss model is:

$$
\begin{aligned}
& L_{\text {pathloss }}=K 1+K 2 \lg d+K 3 \lg \left(h_{t e}\right)+K 5 \lg h_{t e} \lg d \\
& =K 1+K 3 \lg \left(h_{t e}\right)+\left(K 5 \lg h_{t e}+K 2\right) \lg d
\end{aligned}
$$

Among them, K2 takes a typical value of 5.83, K5 takes a typical value -6.55 .

The purpose of parameter correction is to use the measured data to fit a linear regression equation, and finally list the regression equation to calculate the $\mathrm{K}$ parameter. The above formula is organized as:

$$
\begin{aligned}
& L_{\text {pathloss }}=K 1+K 3 \lg \left(h_{t e}\right)+\left(K 2+K 5 \lg h_{t e}\right) \lg d \\
& =a+b \lg d \\
& =a+b x
\end{aligned}
$$

\section{2 SPM propagation model optimization process}

(1) LTE outdoor downlink budget method

The focus is on the path loss when the TD-LTE system signals are propagated in the downlink, that is, the downlink budget. Downlink loss formula:

$$
L_{R S R P}=P_{t e}-L_{f}+G_{t e}+G_{r e}-M_{f}-M_{I}-L_{P}-L_{b}-S_{u e}
$$

Table 3 Meaning of each parameter of the downlink

\begin{tabular}{|l|l|l|}
\hline Parameter & Meaning & Default value \\
\hline Body loss & Loss when signal penetrates human body & $0 \mathrm{~dB}$ \\
\hline UE antenna gain & Receiver antenna gain & $0 \mathrm{dBi}$ \\
\hline $\begin{array}{l}\text { Base station } \\
\text { antenna gain }\end{array}$ & Transmit antenna gain & $18 \mathrm{dBi}$ \\
\hline Feeder loss & $\begin{array}{l}\text { Loss of all cables and connectors from the set top to the } \\
\text { transmitting antenna }\end{array}$ & $1-4 \mathrm{~dB}$ \\
\hline Penetration loss & Signal loss through buildings, cars, ships, etc. & $10-20 \mathrm{~dB}$ \\
\hline $\begin{array}{l}\text { Shadow fade } \\
\text { margin }\end{array}$ & $\begin{array}{l}\text { A method to allow for a certain margin considering the } \\
\text { effects of shadow effects }\end{array}$ & - \\
\hline $\begin{array}{l}\text { Receiver } \\
\text { sensitivity }\end{array}$ & \begin{tabular}{l} 
Lowest level to ensure the receiver can work normally \\
\hline $\begin{array}{l}\text { Interference } \\
\text { margin }\end{array}$
\end{tabular} & $\begin{array}{l}\text { In the actual propagation environment, the signal of the } \\
\text { mobile receiver will be affected by its Interference of other } \\
\text { signals, the margin value reserved for this purpose is the } \\
\text { interference margin the amount }\end{array}$ \\
\hline $\begin{array}{l}\text { Base station } \\
\text { transmit power }\end{array}$ & Transmitting antenna power & $-106 \mathrm{dBm}$ \\
\hline
\end{tabular}

Except for the factors that cause the signal power to change due to the LTE system settings, the losses caused by radio waves during space propagation are collectively referred to as path loss $L_{\text {pathloss }}$, so the above link formula is converted into:

$$
L_{R S R P}=P_{t e}-L_{f}+G_{t e}+G_{r e}-L_{\text {pathloss }}
$$

According to the actual measured base station data, where: $L_{f}=6 \mathrm{dBm}, G_{t e}=8 \mathrm{dBm}, G_{r e}=0$, the measured path loss:

$$
L_{\text {pathloss }}=-L_{R S R P}+P_{t e}+2
$$

The base station transmit power $P_{t e}$ depends on the actual parameters of the base station; the mobile station height is $1.5 \mathrm{~m}$; distance: the distance is calculated. According to the GPS geographical coordinates of each sampling point, the straight line distance from each base station in the serving cell can be calculated, and finally 
the distance the logarithmic value of $\operatorname{lgd}$ (the unit of $\mathrm{d}$ is $\mathrm{m}$ ). The nature of the model correction is to fit a regression curve that conforms to the actual value, to obtain the corrected model coefficient, and to substitute the corrected model coefficient into the calculated predicted value. The error should be minimized. At present, the commonly used algorithm for model correction is the least square method, which is also commonly used by network planning simulation software.

The weighted least squares method is to weight the original basic model to make it a new model without heteroscedasticity, that is, to give each data a different weighting value $\omega_{i}$ when the data is fitted, and then use the ordinary minimum The square method estimates its parameters so that the sum of the squares of the deviations between the measured and predicted values reaches a minimum.

Let the plane be fitted with a straight line. The theoretical straight line is $\mathrm{Y}=\mathrm{a}+\mathrm{bx}$. There are $\mathrm{n}$ sets of measured data $\left(x_{i}, y_{i}\right)(\mathrm{i}=1,2, \ldots . ., \mathrm{n})$ that are linearly distributed on the plane. The expression of the weighted least squares method is as follows:

$$
J=\sum_{i=1}^{n} \omega_{i} \delta_{i}^{2}=\sum_{i=1}^{n} \omega_{i}\left[y_{i}-\overline{y_{i}}\right]^{2}
$$

Among them, $\mathbf{J}$ represents the weighted sum of squared deviations, $y_{i}$ is the actual value, and $\bar{y}_{i}$ is the predicted value. The values of the coefficients $a$ and $b$ are obtained using the Lagrange multiplier method.

\subsection{Visualization of wireless communication propagation prediction based on GIS}

Combining GIS technology with mobile communication signal propagation technology, performing path loss calculation of signals transmitted by communication base stations in the study area, and finally obtaining pointby-point signal coverage and signal strength in the study area, thereby realizing visualization and prediction of mobile communication signal propagation and simulation. Specific visualization methods, using ArcGIS spatial analysis tools, based on the GIS model to calculate the communication signal coverage and signal intensity of each point in the study area; when restricted by the regional terrain conditions, the calculation conditions of this model cannot be applied, the application of visual field analysis and electromagnetic waves The free space transmission loss formula supplements the area coverage, that is, the electromagnetic wave free space propagation loss formula is used to calculate the communication loss in the base station point's passable sight. The specific flowchart is shown in Figure 1.

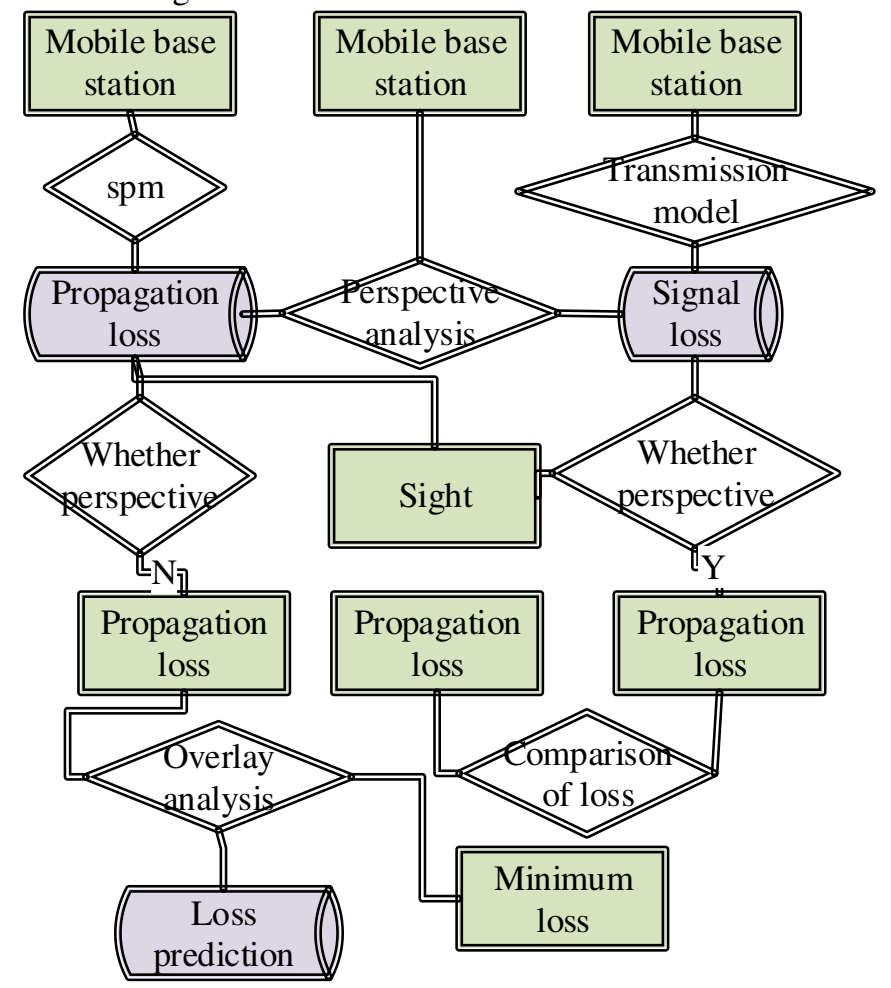

Figure 1 Visual simulation flow of wireless communication network propagation loss prediction 
Create a point feature SHP file. Based on the actual geographic coordinates of each base station, the data of 10 experimental base stations were imported into ARCGIS and the base station layer was formed with the DEM topographic map superimposed on the building height, as shown in Figure 2 below.

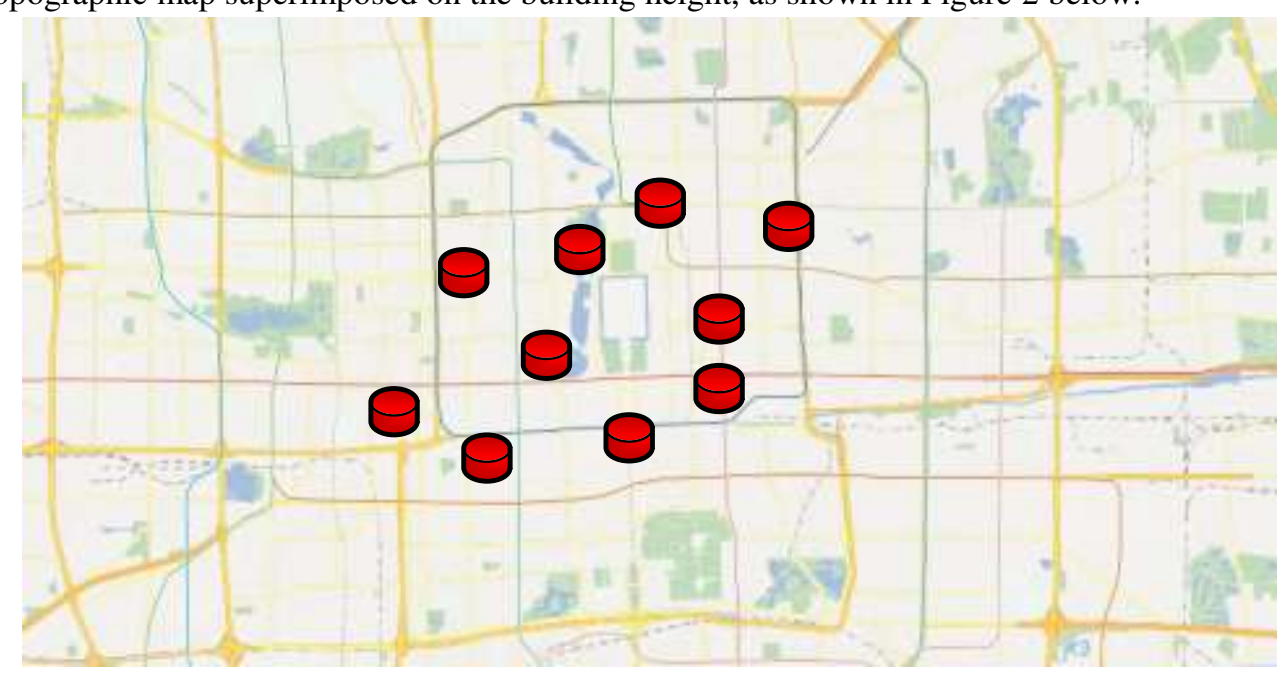

Figure 2 Overlay of experimental base station and DEM

The field of view analysis should be combined with the parameters of the base station, such as the height of the base station, the azimuth of the base station, the mechanical downtilt of the base station, and the coverage radius of the base station. Therefore, according to actual needs, add some fields to the attribute table, some of the data are shown in Table 4 below.

Table 4 Fields added in the base station of the visible domain

\begin{tabular}{|l|l|l|l|l|}
\hline OFFSETA & VERT2 & AZIMUTH1 & AZIMUTH2 & RADIUS2 \\
\hline 15 & 13 & 0 & 65 & 2000 \\
\hline 15 & 12 & 60 & 256 & 2000 \\
\hline 16 & 12 & 252 & 345 & 2000 \\
\hline 24 & 13 & 0 & 35 & 2000 \\
\hline 25 & 6 & 32 & 165 & 2000 \\
\hline 25 & 8 & 162 & 270 & 2000 \\
\hline 27 & 13.78 & 0 & 25 & 2000 \\
\hline
\end{tabular}

\section{Results and Discussion}

The single-length path of the measured data is about 23 kilometers, but in order to obtain more data, each base station repeats the test 4 times. The measured data points are 16,144. The segmented statistics of RSRP level signal values are shown in Table 5, where The minimum value is $-109 \mathrm{dBm}$, the maximum value is -52 $\mathrm{dBm}$, and the average value is $-78 \mathrm{dBm}$.

Table 5 RSRP level signal segment statistics

\begin{tabular}{|l|l|l|l|l|}
\hline \multicolumn{5}{|c|}{ RSRP } \\
\hline Order & Range & Samples & PDF & CDF \\
\hline 1 & {$[-$ inf,-130] } & 0 & $0 \%$ & $0 \%$ \\
\hline 2 & {$[-130,-105]$} & 16 & $0.2 \%$ & $0.15 \%$ \\
\hline 3 & {$[-105,-90]$} & 512 & $4.17 \%$ & $3.29 \%$ \\
\hline 4 & {$[-90,-80]$} & 5233 & $32.34 \%$ & $35.89 \%$ \\
\hline 5 & {$[-80,-76]$} & 7089 & $43.8 \%$ & $79.68 \%$ \\
\hline 6 & {$[-76$, inf $]$} & 3297 & $20.5 \%$ & $100 \%$ \\
\hline Total & 16133 & & Average & -68.97 \\
\hline Maximum & -53 & & Minimum & -108 \\
\hline
\end{tabular}

According to the $\mathrm{K}$ parameter correction, $\mathrm{K} 3=5.83, \mathrm{~K} 5=-6.55$ take the default values to correct $\mathrm{K} 1$, $\mathrm{K} 2$. After the SPM model is corrected and the data is preprocessed, the weighted least squares method is used to calculate the linear regression equations of the 3 base stations as shown in Figure 3 below: 


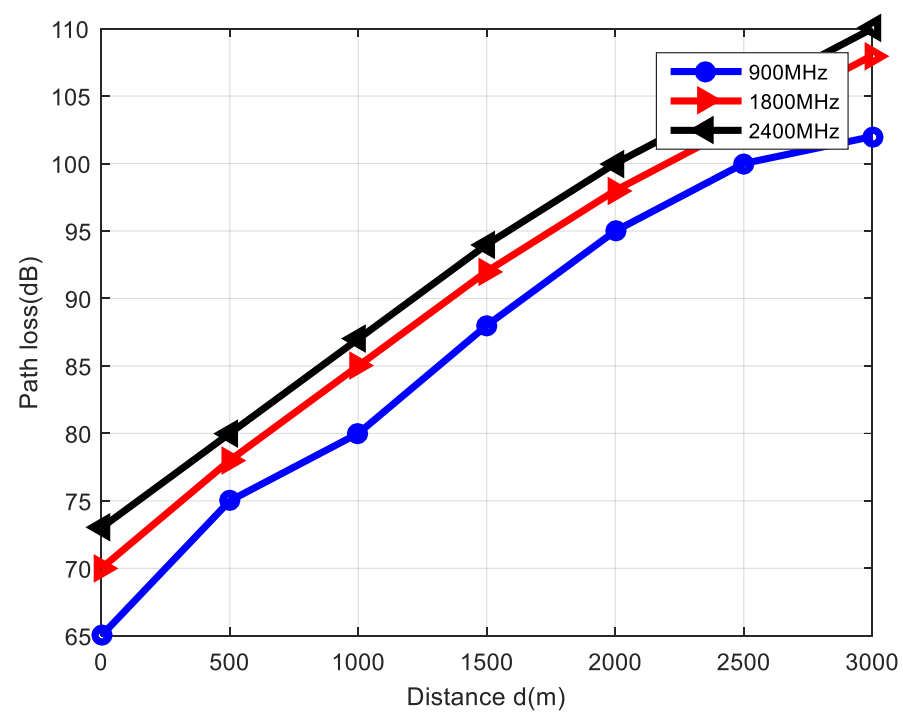

Figure 3 Comparison of the propagation loss of the linear regression correction model of each base station The effective height hte of the test base station antenna is known. Substituting the a and $b$ values of the regression equation into the formula, the $\mathrm{K} 1$ and $\mathrm{K} 2$ values of the test base stations are shown in Table 6 below:

Table 6 Values of the test base stations

\begin{tabular}{|l|l|l|}
\hline Base station & $\mathrm{K} 1$ & $\mathrm{~K} 2$ \\
\hline Yihao & 33.67 & 43.72 \\
\hline Shenlian Electronics & 33.76 & 43.11 \\
\hline Tong He Restaurant & 36.54 & 42.07 \\
\hline Hele New Village & 48.91 & 38.95 \\
\hline Jingji Power Station Trailer & 38.25 & 42.98 \\
\hline Rongde Nonferrous New Materials & 41.61 & 41.26 \\
\hline East District of Hele New Village & 34.26 & 46.56 \\
\hline Dormitory building of Zhongshenglong Electronics Co., Ltd. & 28.33 & 44.69 \\
\hline Zhongshenglong Electronics Co., Ltd. & 27.38 & 46.1 \\
\hline River Tungsten Tyco Landscape Tower & 29.25 & 45.01 \\
\hline Beyond Technology Landscape Tower & 34.42 & 42.44 \\
\hline Yellow Sand D & 28.89 & 43.91 \\
\hline Hele New Village (Hualong Mobile Supermarket) & 37.61 \\
\hline
\end{tabular}

This paper uses the weighted least squares method to analyze the linear regression equation and obtain the prediction improvement model. However, after the model correction is completed, it is necessary to verify whether the model meets the engineering requirements. The actual loss fitting effect after the correction of each base station is shown in Figure 4. 


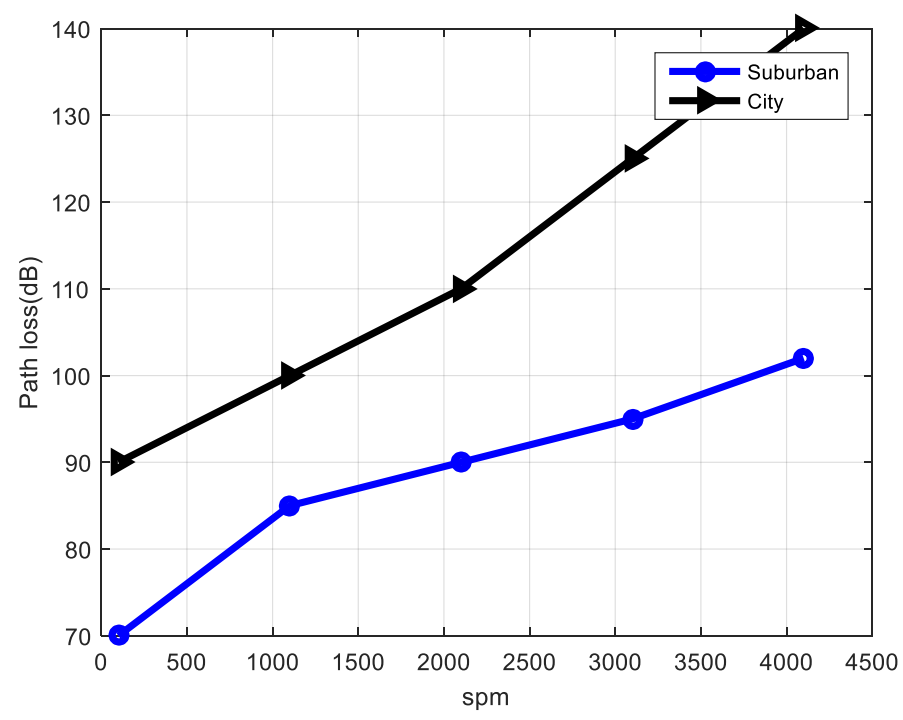

Figure 4 Actual loss prediction chart

TD-LTE network wireless signal effective flow product is 20.38 square kilometers, within this effective range, 298 distribution network communication points were selected for signal strength testing. The test results are shown in Figure 5. The test results show that $79 \%$ of the distribution points have good signals (RSRP> $115 \mathrm{dBm})$ and $15 \%$ have poor signals $(-115 \mathrm{dBm}\langle\mathrm{RSRP}\rangle-125 \mathrm{dBm})$.

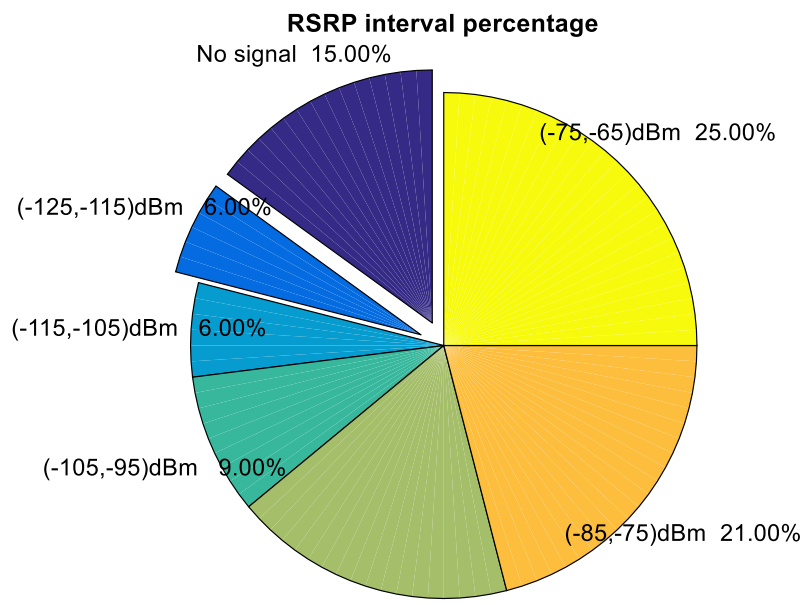

$(-95,-85) \mathrm{dBm} \quad 18.00 \%$

Figure 5 RSRP values of 298 distribution points in the TD-LTE signal coverage area

As above, the weighted least squares method was used to correct the SPM models of the base stations, and an improved model applicable to each base station was obtained. The corrected K1, K: parameters are within the reference range and meet the model requirements The final parameter verification results show that the average and variance of the actual and predicted values meet the evaluation requirements, and the model correction results are good.

\section{Conclusion}

The key technologies of each part of the TD-LTE wireless network planning process are studied. Including the determination of planning goals, the analysis of propagation models, the estimation of coverage and capacity, the analysis and design of wireless network parameters, and the design of the simulation process, the focus of which is the estimation of coverage and capacity, the TD-LTE wireless network planning is constructed in detail Coverage and capacity estimation. According to the theoretical analysis, the general algorithm and basic procedure of model correction are designed, and the K parameters suitable for the TD-LTE system in the study area are obtained by fitting. The result is that the propagation prediction model improved by this method is close to the measured data, and has a certain practical reference value.

\section{List of Abbreviations}


GIS: Geographic Information System

SPM : Standardn Propagation Model

TD-LTE: Time Division Long Term Evolution

GSM: Global System for Mobile Communications

CDMA: Code Division Multiple Access

LTE: Long Term Evolution

GPS: Global Positioning System

RMS: Root Mean Square

RSPR: Reference Signal Receiving Power

\section{Acknowledgements}

The author would like to express her sincere thanks to the referees for the careful reading and their important comments which helped improve the original paper.

\section{Competing interests:}

The authors declare that they have no competing interests

\section{Availability of data and materials}

Not applicable.

\section{Funding:}

Not applicable

\section{References}

[1] Ma L, Hu S, Min Q, et al. (2017). Energy Consumption Optimization of High Sulfur Natural Gas Purification Plant Based on Back Propagation Neural Network and Genetic Algorithms [J]. Energy Procedia, 105:5166-5171.

[2] Deussom Djomadji, Tonye Emmanuel. (2015). New Propagation Model Optimization Approach based on Particles Swarm Optimization Algorithm[J]. International Journal of Computer Applications, 118(10):9758887.

[3] Li T, Ying S, He P. (2016). Research on the Material Mechanics Performance Prediction Based on Back Propagation Neural Network[J]. Journal of Computational \& Theoretical Nanoscience, 13(4):2519-2523.

[4] Wei, Jinling, Yu, Xueyong, Wang, Yingbo. (2015). The Optimization Model Based on Traffic Flow and Safety[J]. Journal of Computational \& Theoretical Nanoscience, 12(12):6265-6271.

[5] Qiang Liu, Xiaoxia Wan, Dehong Xie. (2014). Optimization of spectral printer modeling based on a modified cellular Yule-Nielsen spectral Neugebauer model[J]. Journal of the Optical Society of America A Optics Image Science \& Vision, 31(6):1284-1294.

[6] Zachaeus Kayode Adeyemo, Owolabi Kehinde Ogunremi, Isaac Akinwale Ojedokun. (2016). Optimization of Okumura-Hata Model for Long Term Evolution Network Deployment in Lagos, Nigeria[J]. International Journal on Communications Antenna \& Propagation, 6(3):146.

[7] Pan J H, Liu X.(2015). Assessment of landscape ecological security and optimization of landscape pattern based on spatial principal component analysis and resistance model in arid inland area: A case study of Ganzhou District, Zhangye City, Northwest China][J]. Ying Yong Sheng Tai Xue Bao, 26(10):3126-3136.

[8] Yang X, Xu G, Qi L, et al. (2017). Authorship attribution of source code by using back propagation neural network based on particle swarm optimization[J]. Plos One, 12(11): 187-204.

[9] Rudy J. M. Geelen, Yingjie Liu, John E. Dolbow. (2018). An optimization - based phase - field method for continuous - discontinuous crack propagation[J]. International Journal for Numerical Methods in Engineering, 116(1), 345-367.

[10] Ye, Yingcong, Sun, Kai, Kuang, Lihua. (2017). Spatial layout optimization of urban space and agricultural space based on spatial decision-making[J]. Transactions of the Chinese Society of Agricultural Engineering, 33(16):256-266.

[11] Fan Gu, Yi Xiong Huang, Chuan Ming Chen.(2017) Construction and optimization of ecological network for nature reserves in Fujian Province, China[J]. Chinese Journal of Applied Ecology, 28(3):1013-1020.

[12] Khamis Naba Sayl, Nur Shazwani Muhammad, (2017). Ahmed El-Shafie. Optimization of area-volumeelevation curve using GIS-SRTM method for rainwater harvesting in arid areas[J]. Environmental Earth Sciences, 76(10):368-378.

[13] Hou C, Xiao Y, Cao Y, et al. (2018). Prediction of synchronous closing time of permanent magnetic actuator for vacuum circuit breaker based on PSO-BP[J]. IEEE Transactions on Dielectrics \& Electrical Insulation, 24(6):3321-3326. 
[14] Alejandro De La Fuente, Guillem Femenias, Felip Riera-Palou. (2018). Subband CQI Feedback-Based Multicast Resource Allocation in MIMO-OFDMA Networks[J]. IEEE Transactions on Broadcasting, 64(4):846-864.

[15] Joan Palacios, Danilo De Donno, Joerg Widmer.(2017). Lightweight and Effective Sector Beam Pattern Synthesis With Uniform Linear Antenna Arrays[J]. IEEE Antennas \& Wireless Propagation Letters, 16(99):605-608.

[16] Giulio Zuccaro, Daniela De Gregorio, Magdalini Titirla. (2018). On the simulation of the seismic energy transmission mechanisms[J]. Ingegneria Sismica, 35(1):567-578.

[17] N. Wang, M. He, H. Wang. (2018). Underwater target feature extraction method based on convolutional neural network[J]. Xi Tong Gong Cheng Yu Dian Zi Ji Shu/Systems Engineering and Electronics, 40(6):1197-1203.

[18] Jinxin Du, Christophe Roblin. (2018). Stochastic Surrogate Models of Deformable Antennas Based on Vector Spherical Harmonics and Polynomial Chaos Expansions: Application to Textile Antennas[J]. IEEE Transactions on Antennas \& Propagation, 66(7):3610-3622.

[19] M. S. Hossain Lipu, M. A. Hannan, Aini Hussain. (2018). State of Charge Estimation for Lithium-ion Battery Using Recurrent NARX Neural Network Model Based Lighting Search Algorithm[J]. IEEE Access, 6(2018):28150-28161.

[20] Shengming Wang, Zhaoyang Hu, Cancan Rong. (2018). Optimization Analysis of Coil Configuration and Circuit Model for Asymmetric Wireless Power Transfer System[J]. Iet Microwaves Antennas \& Propagation, 12(7):1132-1139.

[21] Shanpu Shen, Chi-Yuk Chiu, Ross D. (2018). Murch. Multiport Pixel Rectenna for Ambient RF Energy Harvesting[J]. IEEE Transactions on Antennas \& Propagation, 66(2):644-656.

[22] X. Sun, J. Kong, Z. Liu. (2018). Middle-term power load forecasting model based on kernel principal component analysis and improved neural network[J]. Nanjing Li Gong Daxue Xuebao/Journal of Nanjing University of Science and Technology, 42(3):259-265.

[23] Yan-Bo Wang, Ding-Ge Chang, Yu-Hang Fan. (2017). Acoustic localization of partial discharge sources in power transformers using a particle-swarm-optimization-route-searching algorithm[J]. IEEE Transactions on Dielectrics \& Electrical Insulation, 24(6):3647-3656.

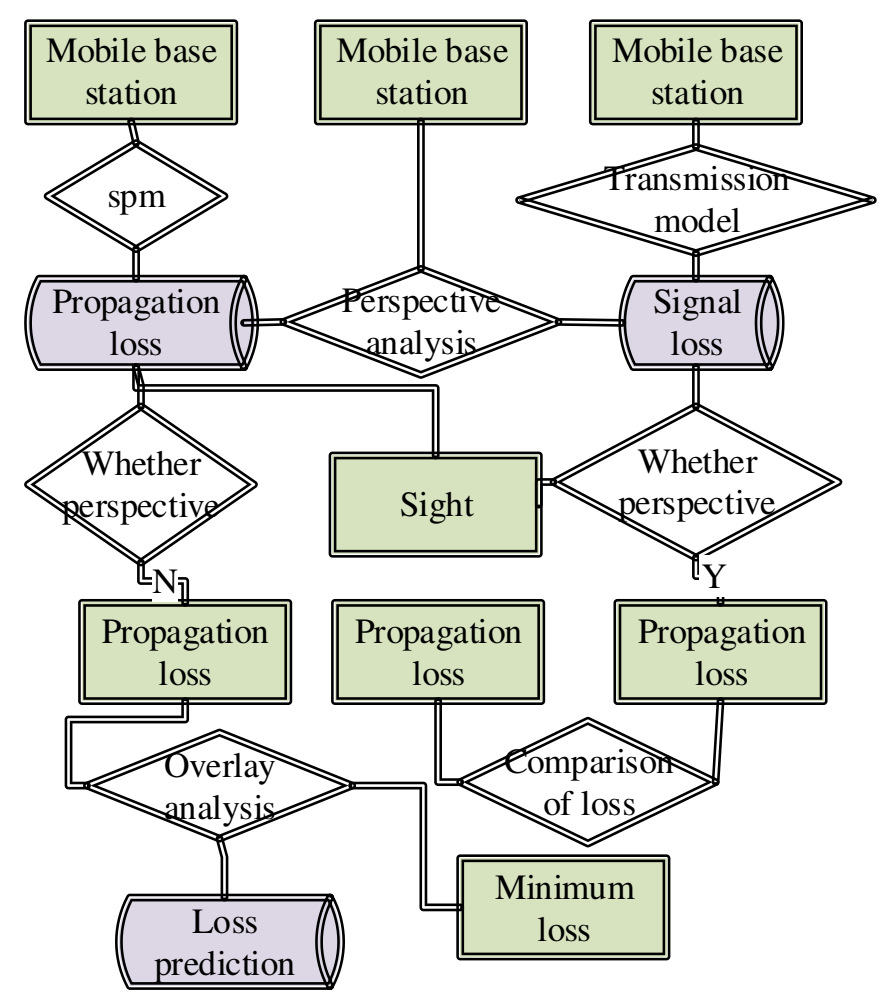

Figure 1 Visual simulation flow of wireless communication network propagation loss prediction 

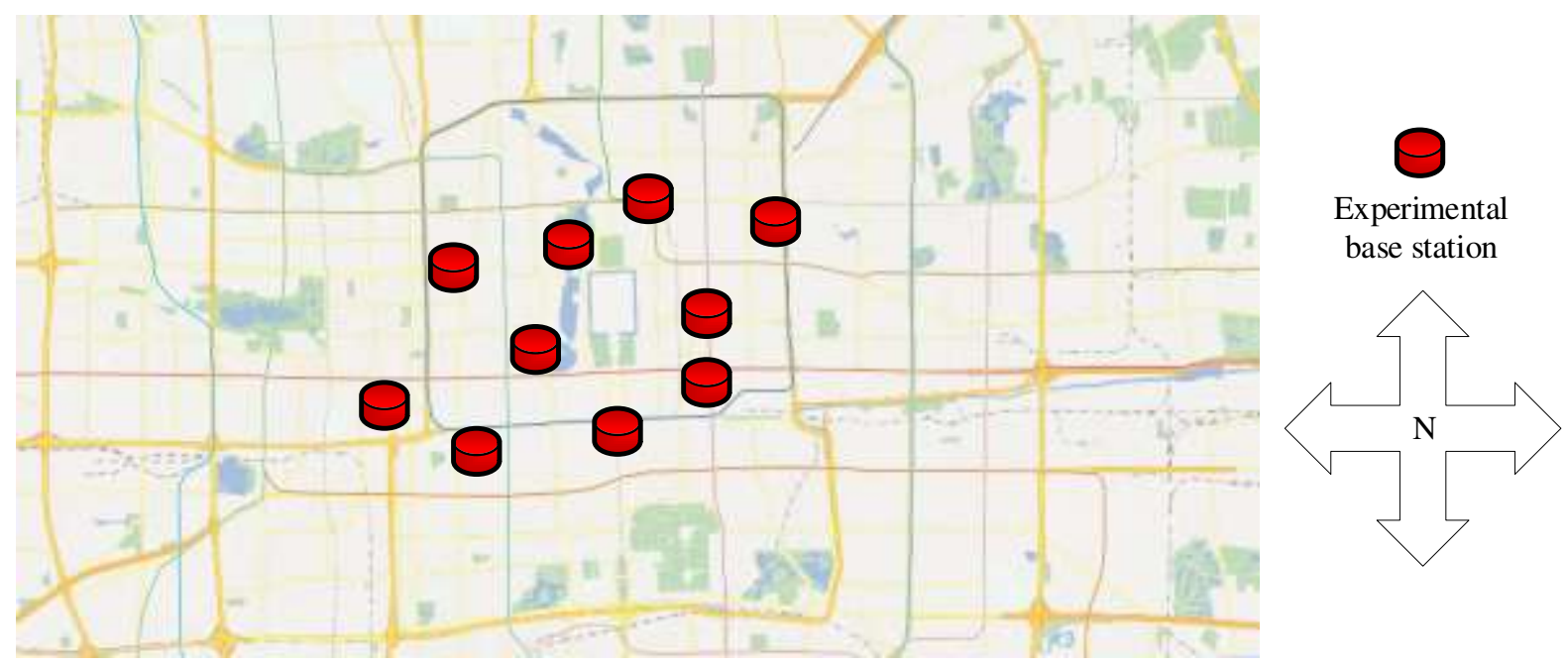

Figure 2 Overlay of experimental base station and DEM

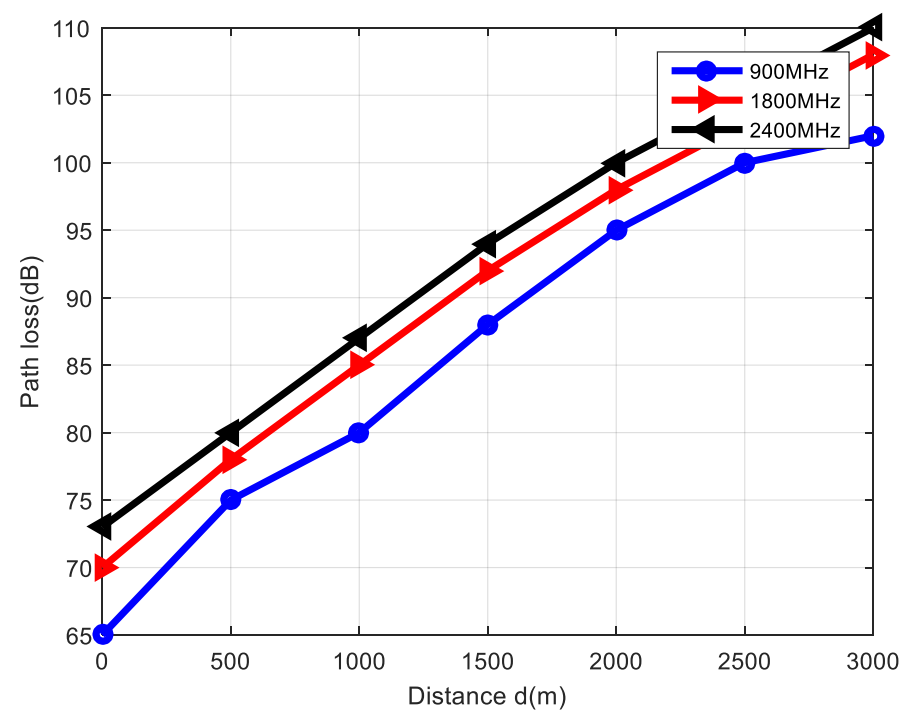

Figure 3 Comparison of the propagation loss of the linear regression correction model of each base station

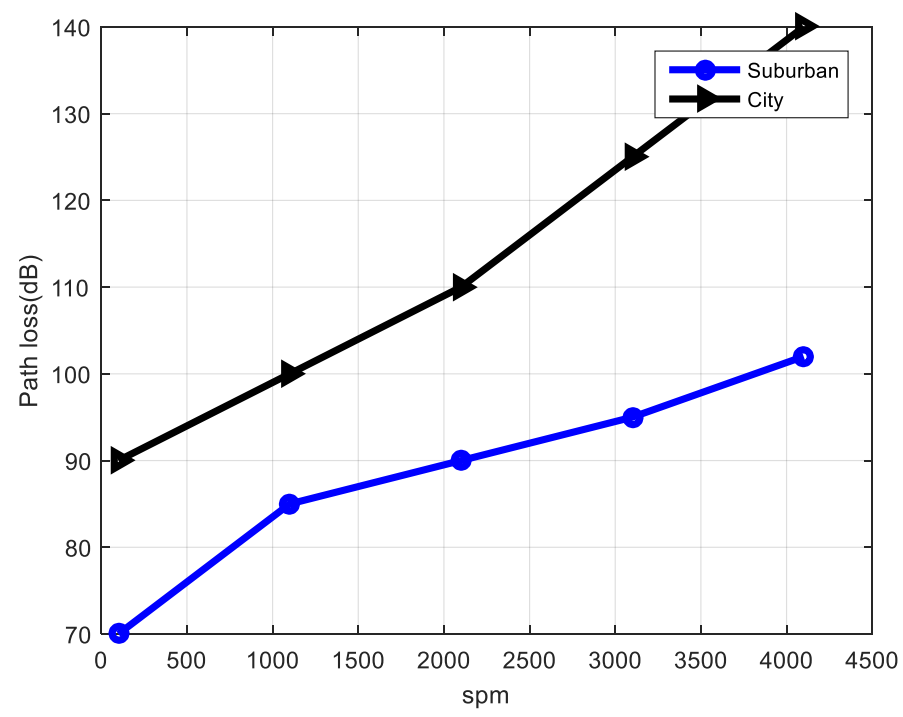

Figure 4 Actual loss prediction chart 


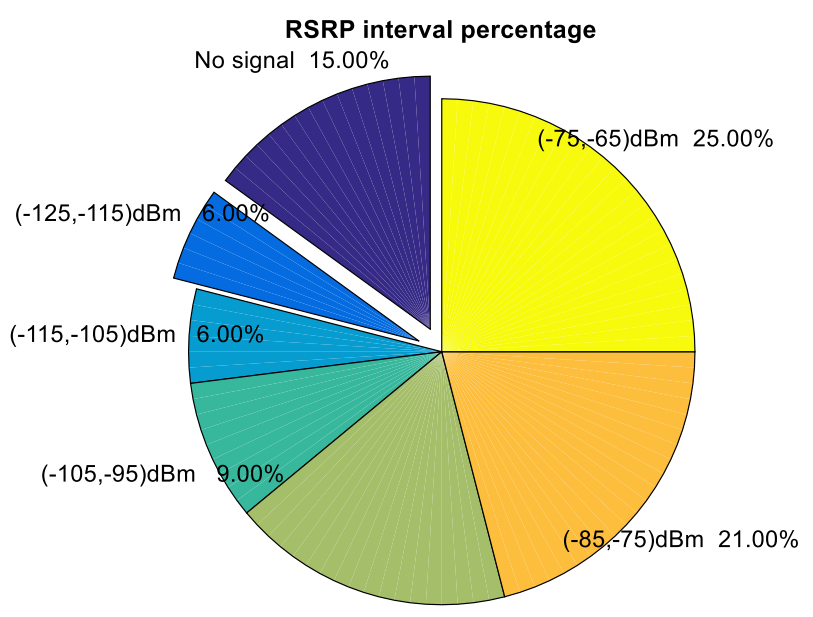

$(-95,-85) \mathrm{dBm} \quad 18.00 \%$

Figure 5 RSRP values of 298 distribution points in the TD-LTE signal coverage area 


\section{Figures}

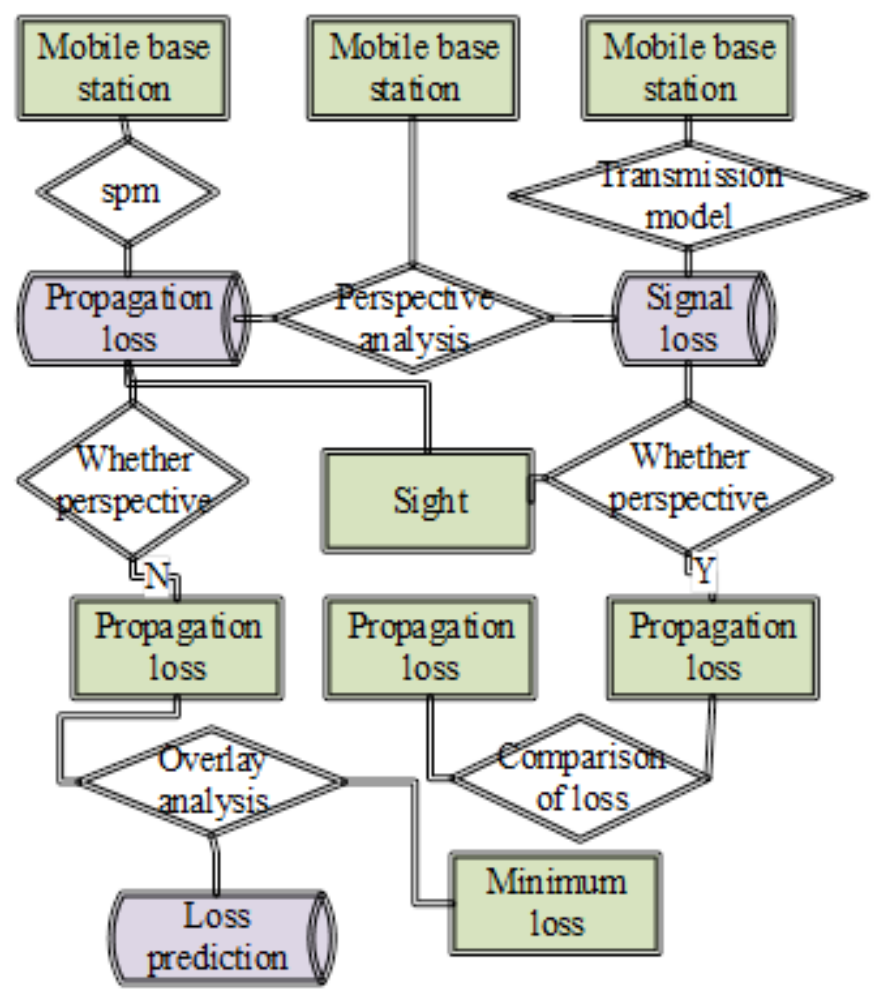

Figure 1

Visual simulation flow of wireless communication network propagation loss prediction
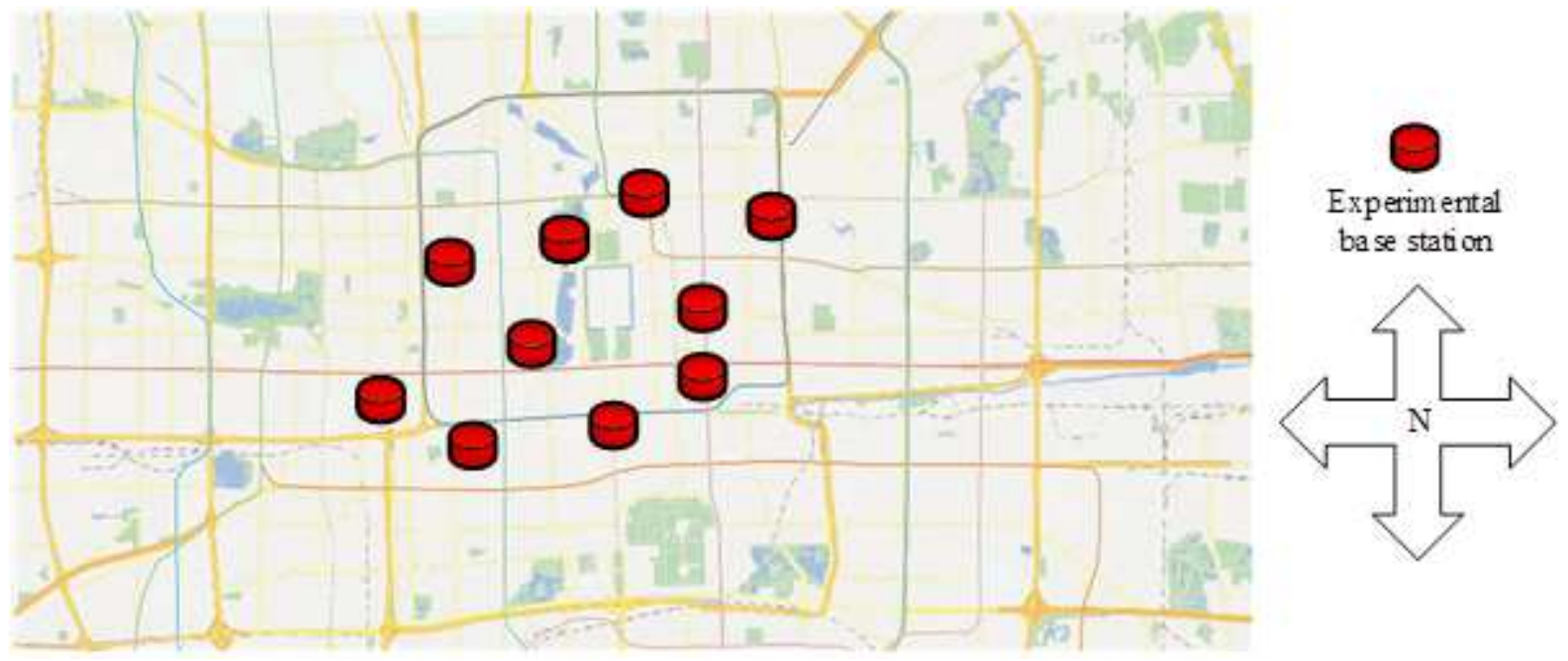

Figure 2

Overlay of experimental base station and DEM Note: The designations employed and the presentation of the material on this map do not imply the expression of any opinion whatsoever on the part of Research Square concerning the legal status of any country, territory, city or area or of its authorities, or concerning the delimitation of its frontiers or boundaries. This map has been provided by the authors. 


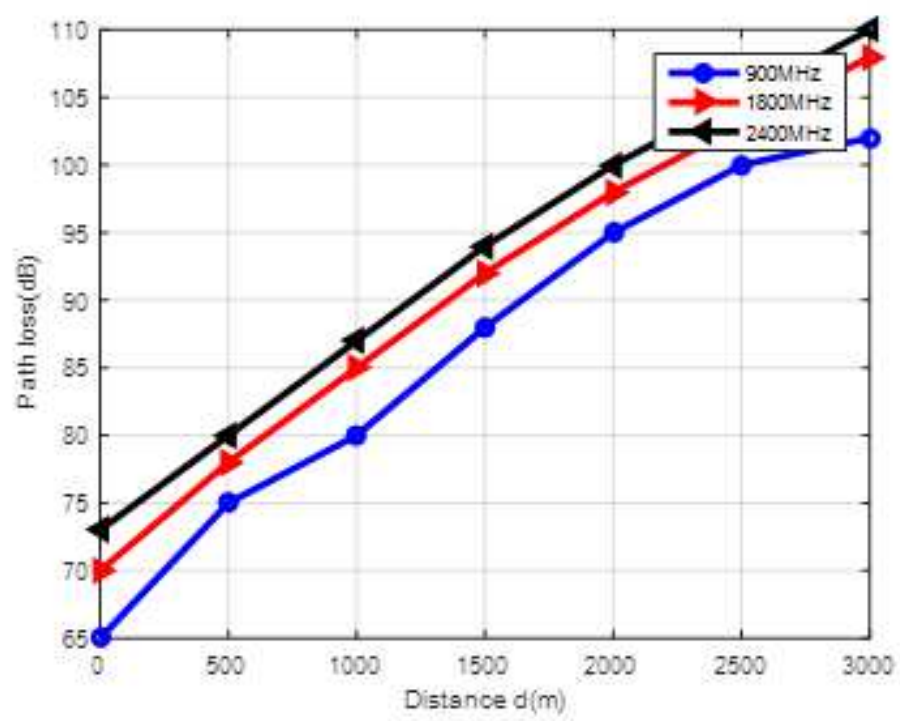

Figure 3

Comparison of the propagation loss of the linear regression correction model of each base station

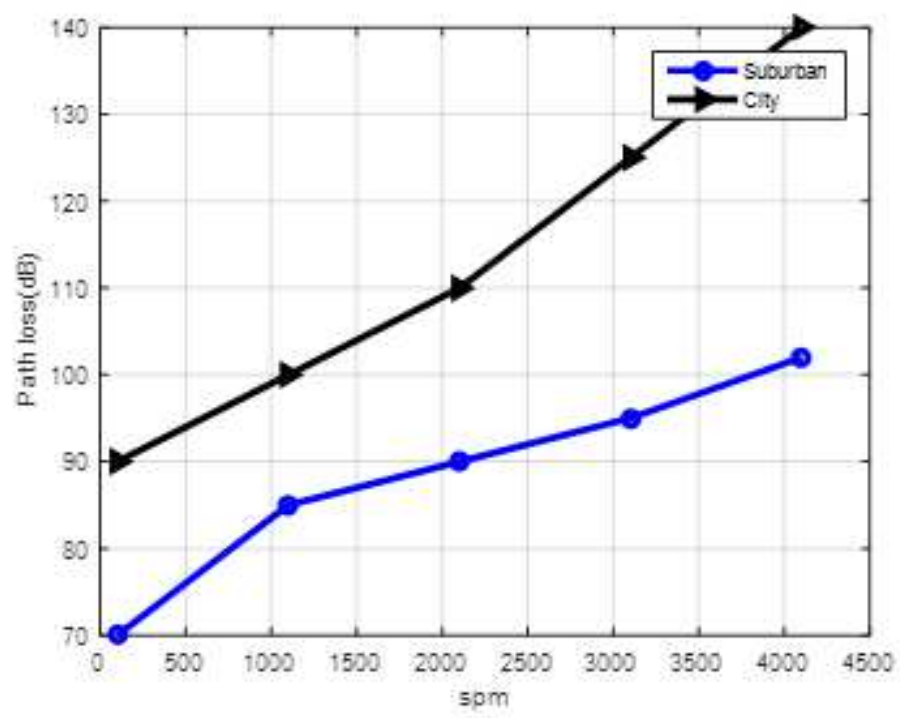

Figure 4

Actual loss prediction chart 


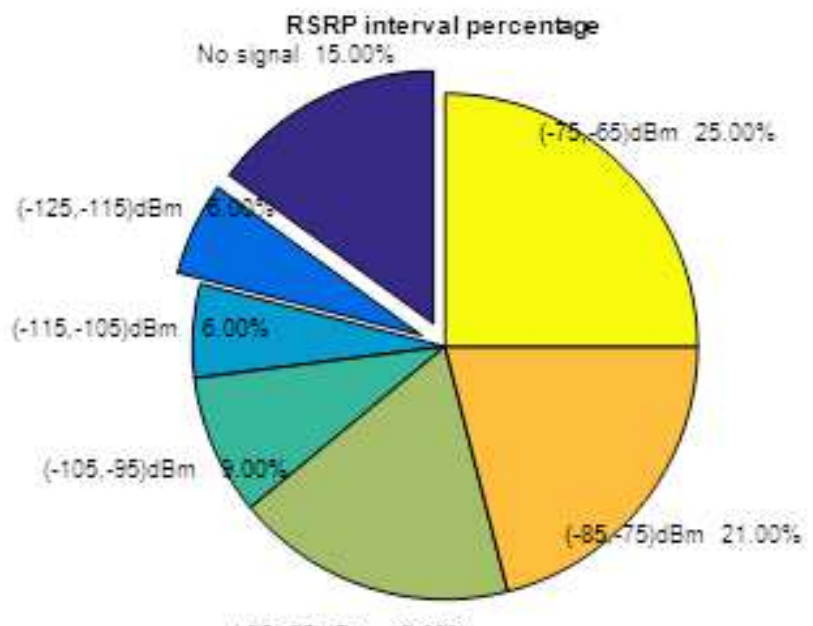

$(-95,-35) 0 \mathrm{Bm} \quad 18.00 \%$

Figure 5

RSRP values of 298 distribution points in the TD-LTE signal coverage area 